University of Nebraska - Lincoln

DigitalCommons@University of Nebraska - Lincoln

December 1972

\title{
Electron Transmission Spectroscopy in Atomic Hydrogen
}

L. Sanche

Department of Engineering and Applied Science, Mason Laboratory, Yale University, New Haven, Connecticut

Paul Burrow

University of Nebraska - Lincoln, pburrow1@unl.edu

Follow this and additional works at: https://digitalcommons.unl.edu/physicsburrow

Part of the Physics Commons

Sanche, L. and Burrow, Paul, "Electron Transmission Spectroscopy in Atomic Hydrogen" (1972). Paul Burrow Publications. 25.

https://digitalcommons.unl.edu/physicsburrow/25

This Article is brought to you for free and open access by the Research Papers in Physics and Astronomy at DigitalCommons@University of Nebraska - Lincoln. It has been accepted for inclusion in Paul Burrow Publications by an authorized administrator of DigitalCommons@University of Nebraska - Lincoln. 


\title{
PHYSICAL REVIEW LETTERS
}

\begin{tabular}{lcc}
\hline \hline Volume 29 & 18 DECEMBER 1972 & Number 25 \\
\hline \hline
\end{tabular}

Electron Transmission Spectroscopy in Atomic Hydrogen*

\author{
L. Sanche $\dagger$ and P. D. Burrow \\ Department of Engineering and Applied Science, Mason Laboratory, \\ Yale University, New Haven, Connecticut 06520
}

(Received 25 October 1972)

\begin{abstract}
An electron transmission experiment is used to study the resonances in the total scattering cross section of atomic hydrogen below the threshold of the first excited state. The three lowest resonances, designated ${ }^{1} S,{ }^{3} P$, and ${ }^{1} D$, are observed and their energies and decay widths are found to be in good agreement with the values predicted theoretically using close coupling with correlation.
\end{abstract}

Electron scattering from atomic hydrogen and the formation of compound states at energies below the first excited state have been the subject of many theoretical studies. ${ }^{1}$ Both the energies and the decay widths of the major resonances have been calculated with high accuracy. Experimental verification of the theories is not easy, however, because of the difficulties encountered in combining monoenergetic electron techniques with the production of atomic hydrogen. This paper presents the results of a transmission experiment in atomic hydrogen which has a high sensitivity for observation of resonances. An analysis of the data confirms with high accuracy the predictions of the theory using close coupling with correlation (ccc) for both the locations and the decay widths of the ${ }^{1} S,{ }^{3} P$, and ${ }^{1} D$ resonances.

There are only three previous experiments dealing with resonances in $\mathrm{H}$ below the first excited state. The first of these, a transmission experiment by Schulz, ${ }^{2}$ revealed the presence of structure in the total scattering cross section but did not resolve separate resonances. Two studies of differential elastic scattering have been made, that of Kleinpoppen and Raible ${ }^{3}$ and the considerably more extensive work of McGowan, Clarke, and Curley, ${ }^{4}$ portions of which were reanalyzed in several subsequent papers. ${ }^{5-7}$ Both studies were carried out at or very near a scattering angle of $90^{\circ}$ and thus information regard- ing $p$-wave structure was suppressed.

In the present experiment, we transmit a monoenergetic electron beam through a collision chamber containing a sufficient density of atomic hydrogen to cause appreciable attenuation of the electron beam. The unscattered transmitted current is related exponentially to the total scattering cross section. Data taken by means of this technique in atomic hydrogen are particularly simple to interpret since the pronounced interference between different partial waves which appears in the differential elastic cross section is absent.

Since the transmission experiment of Schulz, ${ }^{2}$ a number of advances have made possible a considerably improved version of this type of experiment. The first of these was the development of the trochoidal monochromator for production of high-resolution electron beams in axial magnetic fields. ${ }^{8}$ The second was the introduction of a sinusoidal modulating voltage applied to the collision region and subsequent detection of the derivative of the transmitted current. This technique, introduced by Sanche and Schulz, ${ }^{9}$ produces an enhancement in the resonance structure in the total cross section. Both of these techniques have been discussed previously in detail.

In transmission experiments, a higher gas density is required than is normally found in wellcollimated atomic beams. Although microwave 


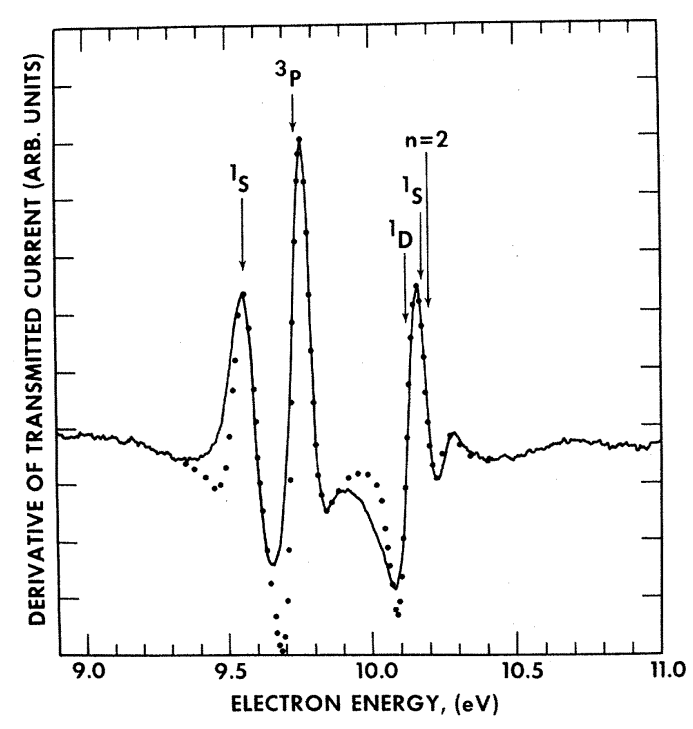

FIG. 1. The solid line shows the derivative of the transmitted current through atomic hydrogen measured as a function of the electron impact energy. The arrows indicate the positions of several resonances as predicted theoretically and the threshold for excitation to the $n=2$ level. The solid circles are the results of an optimum fit to the experimental data from which the resonance parameters were derived.

breakdown of hydrogen contained in Pyrex tubing is known to produce atom densities suitable for electron beam attenuation, introduction of the metal electrodes necessary to define the collision region in the flowing gas causes considerable atomic recombination. We have chosen an intermediate approach used previously in a study of the excitation of atomic hydrogen near threshold by electron impact. ${ }^{10} \mathrm{~A}$ microwave cavity surrounding Pyrex tubing is used to produce dissociation. After flowing a few centimeters, the gas effuses through a slit in the end of the tubing and passes into the collision chamber which is pumped at high speed. To achieve high gas densities, the electron beam, which travels parallel to the long dimension of the slit, intersects the gas jet $1.5 \mathrm{~cm}$ from the slit.

The solid line in Fig. 1 shows a plot of the derivative of the transmitted current measured as a function of the electron impact energy. The location and designation of several resonances and the threshold for excitation to the $n=2$ level are indicated by arrows. The resonance energies are the ccc values of Burke. ${ }^{1}$ The solid circles in Fig. 1 show a fit to the experimental results which was calculated in the following manner: Each important resonance was represented by a
Breit-Wigner single-level profile. The background phase shift in each of the relevant partial waves, which determines the characteristic shape of each resonance, has been calculated with great accuracy by variational techniques at energies somewhat below the resonances, and we have extrapolated these into the region of interest. ${ }^{11}$ The transmitted current was calculated including the effect of Doppler broadening and approximating the electron energy distribution with a Gaussian profile. Finally, the derivative of the transmitted current was computed. ${ }^{12}$

The effective width of the electron distribution was determined by a fit to the width of the ${ }^{3} P$ peak in Fig. 1. This resonance is considerably narrower than the electron distribution, and thus the width of the ${ }^{3} P$ structure in Fig. 1 is determined by instrumental broadening. This led to a distribution having a full width at half-maximum of $70 \mathrm{meV}$ and is in approximate agreement with the width measured from a retarding curve ( 40 $\mathrm{meV}$ ) plus the broadening produced by the modulation voltage $(40 \mathrm{meV}) .^{13}$

With the electron energy distribution thus specified, the computed peak heights in the derivative are primarily determined by the decay widths of the resonances. Because the results of the computation must be normalized to the experimental data at one of the peaks, it is not possible to determine the decay width of the corresponding resonance. Fortunately, the data of McGowan, Clarke, and Curley ${ }^{4}$ were extensively analyzed ${ }^{6}$ to deduce the characteristics of the lowest ${ }^{1} S$ resonance, and the decay width appears to be in agreement with ccc theory. We have chosen therefore to normalize the computed derivative of the transmitted current to the experimental data at the ${ }^{1} S$ peak using the theoretical decay width of 0.0475 $\mathrm{eV}$. Because of the large width of this resonance, the peak height in the derivative does not vary rapidly with the choice of decay width. We have varied the width between the limits found by McGowan ${ }^{6}$ and incorporate this uncertainty into the error limits of the other resonance widths. A fit to the remaining experimental data was then made by varying the resonance energies and decay widths. The results which gave the best ag reement with the experiment are indicated by the solid circles in Fig. 1, and the optimum parameters are tabulated in Table $I$.

The sharp dip and peak in the derivative just above the $n=2$ threshold cannot be accounted for by a resonance below threshold. The cross section for excitation to the $n=2$ levels is known to 
TABLE I. Resonance energies $\left(E_{R}\right)$ and decay widths $(\Gamma)$ of atomic hydrogen (eV).

\begin{tabular}{cccccrrr}
\hline & \multicolumn{2}{c}{$\begin{array}{c}\text { Experiment } \\
\text { McGowan }^{\mathrm{a}}\end{array}$} & \multicolumn{2}{c}{$\begin{array}{c}\text { Theory } \\
\text { Burke b }^{\mathrm{b}}\end{array}$} & \multicolumn{2}{c}{$\begin{array}{c}\text { Experiment } \\
\text { Present work }\end{array}$} \\
Resonance & $E_{R}$ & $\Gamma$ & $E_{R}$ & $\Gamma$ & \multicolumn{1}{c}{$E_{R}$} & $\Gamma$ \\
\hline${ }^{1} S$ & 9.56 & 0.043 & 9.560 & 0.0475 & 9.558 & $\ldots$ \\
& \pm 0.010 & \pm 0.006 & & & \pm 0.010 & \\
${ }^{3} P$ & 9.71 & $>0.009$ & 9.740 & 0.00594 & 9.738 & 0.0056 \\
& \pm 0.03 & & & & \pm 0.010 & \pm 0.0005 \\
${ }^{1} D$ & 10.13 & $\ldots$ & 10.125 & 0.0088 & 10.128 & 0.0073 \\
& \pm 0.015 & & & & \pm 0.010 & \pm 0.002 \\
\hline \hline
\end{tabular}

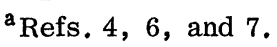

be finite at threshold and to possess a sharp shape resonance at $10.222 \mathrm{eV} .{ }^{14}$ It was necessary to include a simulation of these features to reproduce the structure in the total scattering cross section in Fig. 1. The shape resonance as calculated by Taylor and Burke ${ }^{14}$ is not well described by a single-level Breit-Wigner profile, however, and as our computer program required an analytic representation, the resonance parameters could not be accurately deduced.

The energy scale is calibrated by two means. The primary reference point is the structure appearing in the derivative of the total cross section due to the abrupt onset of excitation to the first excited state at $10.204 \mathrm{eV}$. For the atomic hydrogen resonances described here, we infer overall errors for the resonance energies of $\pm 0.010 \mathrm{eV}$. The secondary calibration is by reference to resonance structure in molecular hydrogen at $11.32 \pm 0.030 \mathrm{eV}$ which is present as a result of incomplete dissociation. This structure had been previously calibrated by Sanche and Schulz ${ }^{9}$ with respect to the $19.34 \pm 0.020-\mathrm{eV}$ resonance of helium in a similar transmission experiment. The two energy scales which we thus obtain differ by only $7 \mathrm{meV}$. The consistency of the energy scales indicates that the absolute error of the calibrations referred to the helium resonance may be considerably smaller than the span of $\pm 0.050 \mathrm{eV}$ given by Sanche and Schulz.

The fit to the experimental data was achieved with considerable accuracy except for the width at half-maximum of the ${ }^{1} S$ peak and for the data at energies between the resonances. Because the ${ }^{1} S$ resonance has a relatively large decay width, as one moves away from the center of the resonance the magnitude of the derivative becomes sensitive to the wings of the electron distribution. The inaccuracy in the fit reflects the approxima- tion that we have used to represent the electron distribution. It is our experience that the trochoidal monochromator produces an electron distribution with wings which do not fall off as rapidly as those in a Gaussian distribution.

The ${ }^{3} P$ resonance was previously observed only as very small structure in the differential elastic scattering by McGowan, Clarke, and Curley. ${ }^{4} \mathrm{Al}-$ though their data were taken at $90^{\circ}$, an angle at which this partial wave is absent, the resonance appeared because of the finite angular resolution of their apparatus. In the present experiment, evaluation of the decay width of the ${ }^{3} P$ resonance is reduced to fitting the ratio of the ${ }^{3} P$ peak height of the derivative to that of the ${ }^{1} S$ resonance. In contrast to the normally used deconvolution techniques, this procedure can be done quite accurately even for small decay widths.

Between $10.125 \mathrm{eV}$ and the $n=2$ threshold at $10.204 \mathrm{eV}$, a great number of resonances are known to exist. However, as demonstrated by Ormonde, McEwen, and McGowen ${ }^{7}$ and confirmed here, the ${ }^{1} D$ resonance predominates. The decay width of this resonance could not be evaluated within such narrow limits as that of the ${ }^{3} P$ resonance. The background phase shift rises rapidly ${ }^{11}$ below the resonance and our extrapolation is more uncertain. The second ${ }^{1} S$ resonance, placed at $10.178 \mathrm{eV}$ theoretically, also contributes a small amount to the signal. A unique fit to the data was therefore not possible.

The energies of the three lowest resonances and the decay widths of the ${ }^{3} P$ and ${ }^{1} D$ states derived from our data are listed in Table $I$. All of the values are seen to be in excellent agreement with Burke's results using the close-coupling theory with correlation.

Thanks are due to G. J. Schulz and Ton That Dinh for many helpful discussions. 
*Work supported by the National Science Foundation. $\dagger$ Present address: Department of Nuclear Medicine, Centre Hospitalier Universitaire, University of Sherbrooke, Sherbrooke, P. Q., Canada.

${ }^{1}$ For a review see P. G. Burke, in Advances in Atomic and Molecular Physics, edited by D. R. Bates and I. Estermann (Academic, New York, 1968), Vol. 4, p. 173.

${ }^{2}$ G. J. Schulz, Phys. Rev. Lett. 13, 583 (1964).

${ }^{3}$ H. Kleinpoppen and V. Raible, Phys. Lett. 18, 24 (1965).

${ }^{4}$ J. W. McGowan, E. M. Clarke, and E. K. Curley, Phys. Rev. Lett. 15, 917 (1965), and 17, 66(E) (1966).

${ }^{5}$ J. W. McGowan, Phys. Rev. Lett. 17, 1207 (1966).

${ }^{6}$ J. W. McGowan, Phys. Rev. 156, 165 (1967).

${ }^{7}$ S. Ormonde, J. McEwen, and J. W. McGowan, Phys. Rev. Lett. 22, 1165 (1969).

${ }^{8}$ A. Stamatovic and G. J. Schulz, Rev. Sci. Instrum. 41,423 (1970).

${ }^{9}$ L. Sanche and G. J. Schulz, Phys. Rev. A $\underline{6}, 69$ (1972), and earlier papers cited in this reference.

${ }^{10}$ P. D. Burrow, in Proceedings of the Seventh International Conference on the Physics of Electronic and Atomic Collisions, Amsterdam, The Netherlands, 1971 (North-Holland, Amsterdam, 1971), p. 316.

${ }^{11}$ The background phase shifts were extrapolated from calculations of C. Schwartz, Phys. Rev. 124, 1468

(1961); R. L. Armstead, Phys. Rev. 171, 91 (1968);
M. K. Gailitis, in Atomic Collisions III, edited by V. Ya. Veldre et al. (Massachusetts Institute of Technology Press, Cambridge, Mass., 1966). The phase in radians for the resonances ${ }^{1} S,{ }^{3} P$, and ${ }^{1} D$ was taken to be $0.88,0.437$, and $0.12-0.16$, respectively.

${ }^{12}$ We are very grateful to Ton That Dinh for the use of his derivative electron transmission program and for a great deal of help in adapting it to this project.

${ }^{13}$ Strictly speaking, this fit is an approximation to the data since a true mathematical derivative with respect to energy was used rather than the change in signal due to the finite modulation voltage. The error is minimized by absorbing additional broadening in the effective electron distribution which is adjusted to fit the measured width of the ${ }^{3} P$ structure. The remaining error, which is comparable to that associated with using a Gaussian approximation for the electron distribution, is neglected compared to the primary source of error, namely, the uncertainty in decay width of the ${ }^{1} S$ resonance to which the other resonances are normalized. We also note that a more elaborate computer fit would allow the extraction of the background phases as well as the parameters found here. The great accuracy with which the phases have been calculated theoretically make this exercise unrewarding.

${ }^{14}$ A. J. Taylor and P. G. Burke, Proc. Phys. Soc., London $\underline{92}, 336$ (1967).

\title{
Zero-Field Level-Crossing Resonances in Optically Pumped $2^{3} S_{1} \mathrm{He}^{4} \dagger$
}

\author{
Robert E. Slocum
}

Texas Instruments Incorporated, Dallas, Texas 75222

(Received 24 October 1972; revised manuscript received 16 November 1972)

Parametric resonances associated with zero-field level crossings of the $2^{3} S_{1}$ level of $\mathrm{He}^{4}$ have been investigated using transverse optical pumping. A steady-state technique based on the $n=0, p=0$ resonance (Hanle effect) is used to measure spin-state lifetimes and determine the dependence of the thermal relaxation time on the state of the active helium discharge. The $n=0, p=1$ resonance is quite strong and suitable for measuring weak magnetic fields. A sensitivity of $1.0 \times 10^{-8} \mathrm{G}$ is demonstrated for this measurement technique.

Resonance effects associated with zero-field level crossings in ground-state or metastable atoms subjected to transverse optical pumping were first reported independently by the author and Reilly ${ }^{1}$ and Lehmann and Cohen-Tannoudji. ${ }^{2}$ In later experiment, Polonsky and Cohen-Tannoudji ${ }^{3}$ utilized transverse optical pumping to observe a series of parametric resonances in $\mathrm{Hg}^{199}$. Recently this technique was used by $\mathrm{Du}-$ pont-Roc, Haroche, and Cohen-Tannoudji ${ }^{4}$ to observe zero-field resonances in $\mathrm{Rb}^{87}$ and measure very weak magnetic fields. ${ }^{5,6}$ In the present Letter, observation of zero-field level-crossing resonances is reported for the $2^{3} S_{1}$ level of $\mathrm{He}^{4}$. Line shapes and amplitudes of the $n=0, p=0$ (Hanle effect ${ }^{7}$ ) and $n=0, p=1$ resonances have been investigated. Two items of particular interest in undertaking these experiments were the suitability of the $n=0, t=1$ resonance for measuring weak magnetic fields and development of a steady-state technique for measuring the spin-state lifetime $\tau_{1}$. This technique would utilize the lifetime relationship with the linewidth $\Delta H=2 / \gamma \tau_{1}$ and would avoid the main difficulties found in applying familiar transient techniques ${ }^{8,9}$ to $2^{3} S_{1}$ helium.

The optical pumping apparatus used in the res- 